\title{
Pericardial tamponade after chronic total occlusion revascularization: a case report and literature review
}

\author{
Jinjing Li ${ }^{1 \#}$, Mingming Zhang ${ }^{1 \#}$, Xiaohui Kang ${ }^{1}$, Ruirui Chen ${ }^{1}$, Fangfang Wang ${ }^{1}$, Wanlin Chen ${ }^{2}$, Xue Li $^{1}$ \\ ${ }^{1}$ Department of Cardiology, Tangdu Hospital, Fourth Military Medical University, Xi'an, China; ${ }^{2}$ Department of Cardiology, People's Hospital of \\ Baoji, Baoji, China \\ \#These authors contributed equally to this work. \\ Correspondence to: Xue Li, MD, PhD. Department of Cardiology, Tangdu Hospital, Fourth Military Medical University, No. 569 Xinsi Road, Xi'an \\ 710038, China. Email: lxhlms@126.com.
}

\begin{abstract}
Pericardial tamponade is a complication of percutaneous coronary intervention (PCI) with extremely high mortality. The rupture of coronary artery causes hypotension and shock, eventually resulting in death due to pericardial tamponade. Because of the complex operation in revascularization of chronic total occlusion (CTO-PCI) lesion, the incidence of pericardial tamponade increases. Usually, we use coronary angiogram to identify the rupture of coronary artery after PCI by the contrast agent. We presented a 67-year-old woman with pericardial tamponade after CTO revascularization. She had chest pain and out of breath for nearly two years. The coronary angiogram showed three branch lesion and CTO lesion of the right coronary artery (RCA). After revascularization of the RCA CTO lesion, the pericardial effusion and low blood pressure occurred, but we didn't find the leak of contrast agents during the final angiography. Then the patient was transferred to cardiac surgery department for emergency thoracotomy. They found the hematoma on the surface of the RCA and finally discharged without any symptoms. Our case approved: (I) there was still the possibility of coronary artery rupture even the coronary angiogram showed no contrast agent leakage from the coronary artery after PCI; (II) the combined use of IVUS and coronary angiogram may improve the accuracy and safety of CTO revascularization procedure.
\end{abstract}

Keywords: Chronic total occlusion (CTO); pericardial tamponade; percutaneous coronary intervention (PCI); coronary angiogram; coronary artery rupture

Submitted Dec 09, 2020. Accepted for publication Apr 07, 2021.

doi: 10.21037/apm-20-2455

View this article at: https://dx.doi.org/10.21037/apm-20-2455

\section{Introduction}

Chronic total occlusion of coronary artery (CTO) refers to a lesion in which the degree of coronary angiography is $100 \%$ occlusion. Numerous observational studies have showed that successful CTO revascularization is associated with better cardiovascular outcomes and enhanced quality of life. PCI is considered as one of the most effective methods of revascularization (1). However, in some cases, PCI could cause severe complication, including contrast induced nephropathy, acute stent thrombosis, stent dislodgement, malignant ventricular arrhythmia and pericardial tamponade $(2,3)$. Usually, we identify vascular rupture by coronary angiogram. But in this case report, we describe an unusual case of pericardial tamponade after CTO revascularization. This patient had pericardial tamponade without contrast agent leakage from coronary artery after PCI procedure. This situation puzzled and confused us.

We present the following article in accordance with the CARE reporting checklist (available at https://dx.doi. org/10.21037/apm-20-2455).

\section{Case presentation}

A 67-year-old woman was admitted to our hospital with paroxysmal chest pain and out of breath for nearly two 


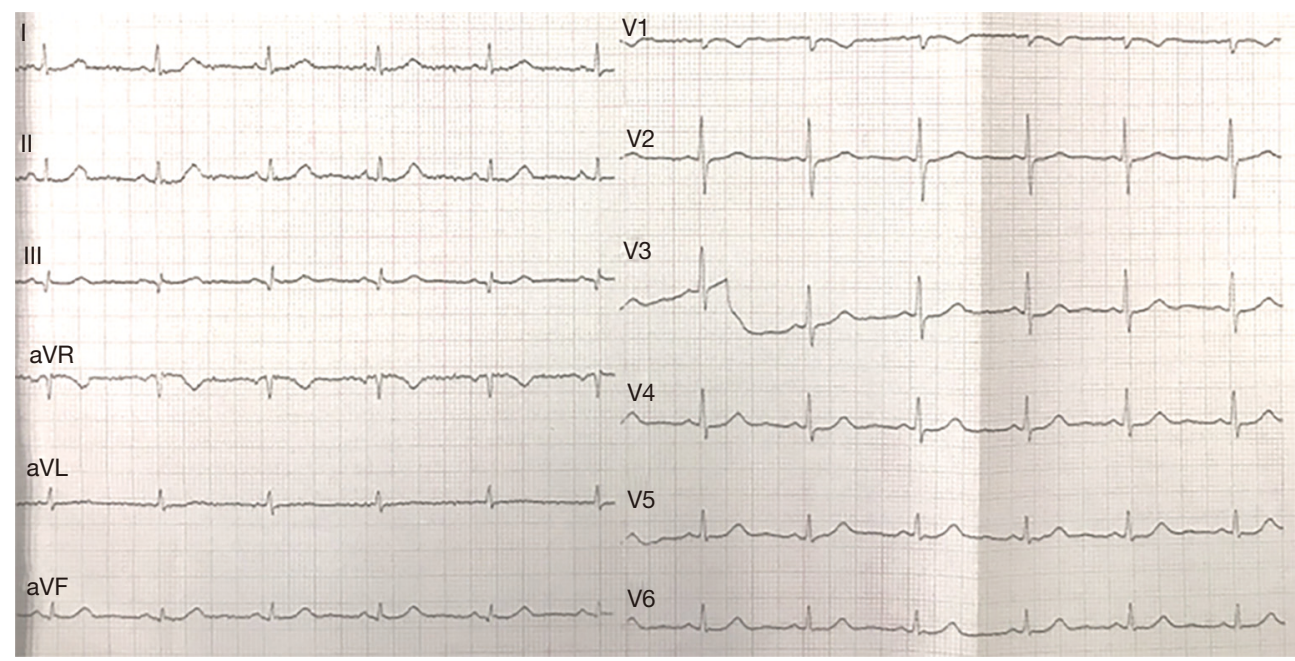

Figure 1 Electrocardiogram (ECG) showed T wave was low in aVL lead.
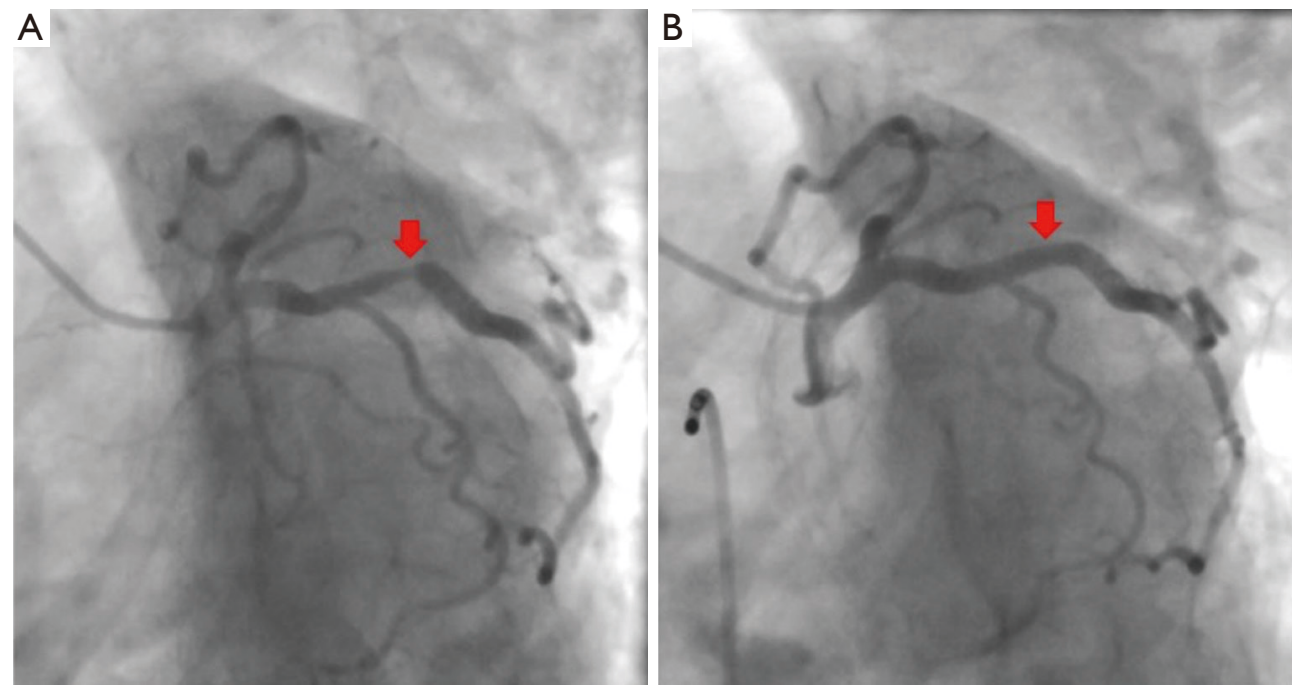

Figure 2 Coronary angiography of the left coronary arteries. (A) The stenosis site of left circumflex artery (LCX, arrowhead). (B) The stenosis site of left circumflex artery (LCX) after percutaneous coronary angioplasty (PCI, arrowhead).

years. The symptoms which often occurred after activity, lasted about 10 minutes and can be relieved by rest or nitroglycerin. She had taken clopidogrel $1(75 \mathrm{mg} / \mathrm{d})$, isosorbide mononitrate $(40 \mathrm{mg} / \mathrm{d})$ and atorvastatin calcium $(20 \mathrm{mg} / \mathrm{d})$. Serum triglyceride level was $3.76 \mathrm{mmol} / \mathrm{L}$. Electrocardiogram (ECG) showed $\mathrm{T}$ wave was low in aVL lead (Figure 1). Echocardiogram displayed that the left atrium was slightly enlarged. Left ventricular ejection fraction (LVEF) was $60 \%$ and there was mild mitral valve insufficiency. Coronary angiogram showed that the right coronary artery (RCA) was completely occluded, and the proximal segment to middle segment of left anterior descending artery (LAD) was occluded about $30-50 \%$, with a length of $50 \mathrm{~mm}$ lesion. The proximal segment of left circumflex artery (LCX) was occluded about $50-85 \%$, with a length of about $18 \mathrm{~mm}$ lesion. We first opened the LCX to alleviate the patient's symptoms by placing a stent (Helios, China; $4.0 \mathrm{~mm} \times 22 \mathrm{~mm}$ ) (Figure 2). The chest pain and out of breath of the patient were relieved significantly after LCX PCI, but there was still chest tightness after severe activity. Compared to preoperation there was no significant difference in ECG (Figure 3). The 


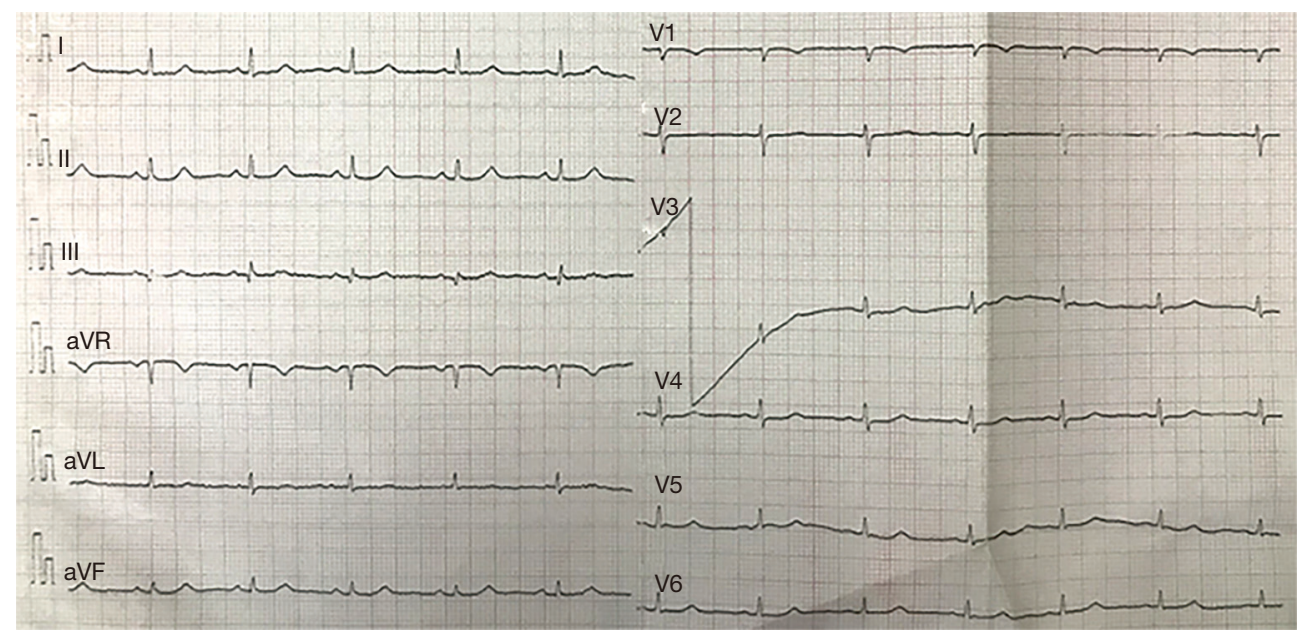

Figure 3 Electrocardiogram (ECG) showed T wave was low in aVL lead.

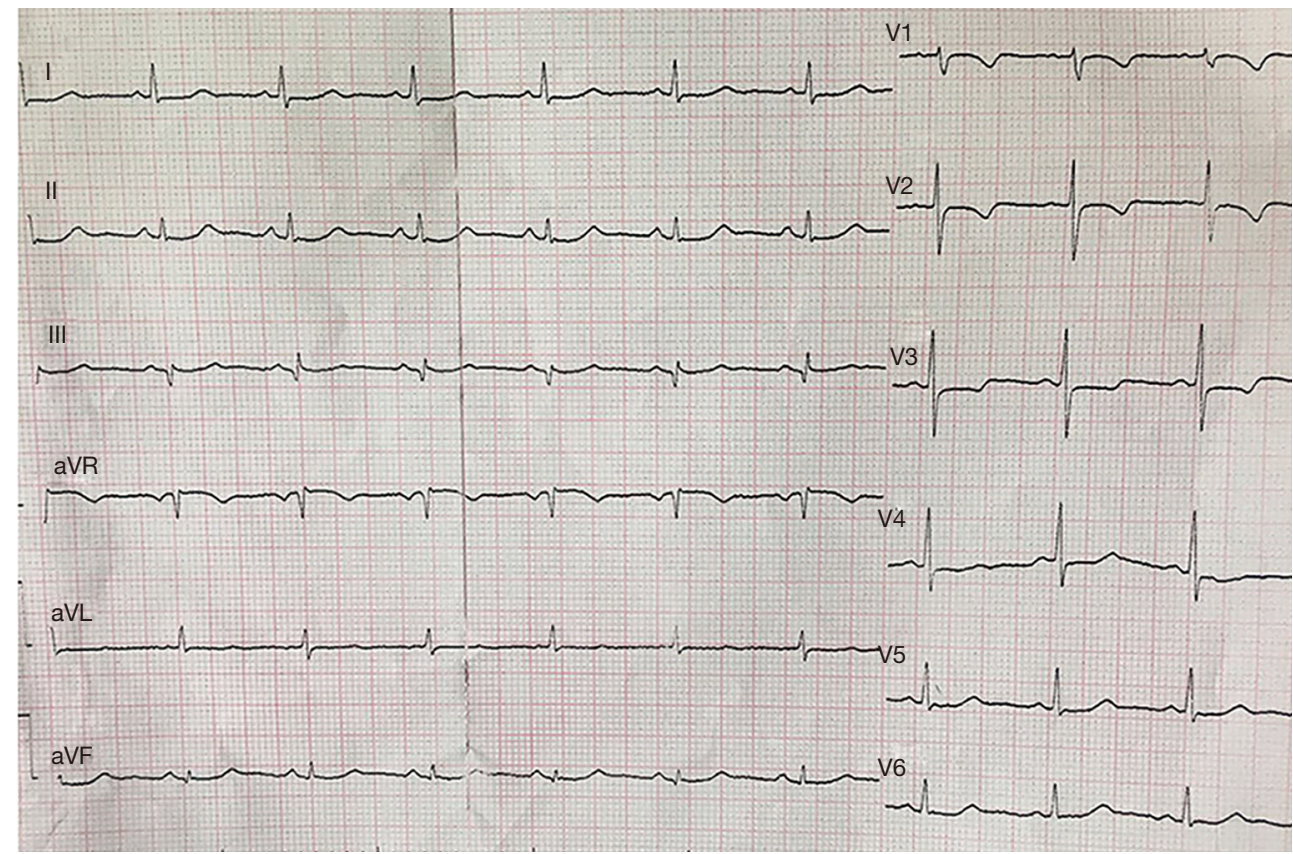

Figure 4 Electrocardiogram (ECG) revealed ST depression and T waves inversion in V1-V4 leads.

patient took aspirin $(100 \mathrm{mg} / \mathrm{d})$, clopidogrel $(75 \mathrm{mg} / \mathrm{d})$ and atorvastatin calcium $(20 \mathrm{mg} / \mathrm{d})$ for postoperative treatment. After 6 weeks, ECG showed that the ST depression and $\mathrm{T}$ inversion in V1-V4 (Figure 4). Therefore, we planned to revascularize the RCA. By SAL 1.0 guiding, we used $\mathrm{XT}-\mathrm{R}$ and Gaia First wire going through the lesion part of RCA. We used $1.25 \mathrm{~mm} \times 10 \mathrm{~mm}$ balloon (Tazuna, Japan), $1.5 \mathrm{~mm} \times 15 \mathrm{~mm}$ balloon (Sprinter, USA) and
$2.0 \mathrm{~mm} \times 20 \mathrm{~mm}$ balloon (Traveler, USA) to expand vascular. We placed $2.25 \mathrm{~mm} \times 8 \mathrm{~mm}$ and $2.5 \mathrm{~mm} \times 28 \mathrm{~mm}$ XIENCE $\mathrm{X}$ stents from distal to proximal by the sequence. Finally, we used $2.5 \mathrm{~mm} \times 15 \mathrm{~mm}$ non-expansive balloon (Sapphire, the Netherlands) to modify stents. Angiography showed that blood flow of RCA was TIMI 3 (Figure 5). When we withdrew Sal 1.0 guiding, the patient was unconscious suddenly. The echocardiogram showed that 

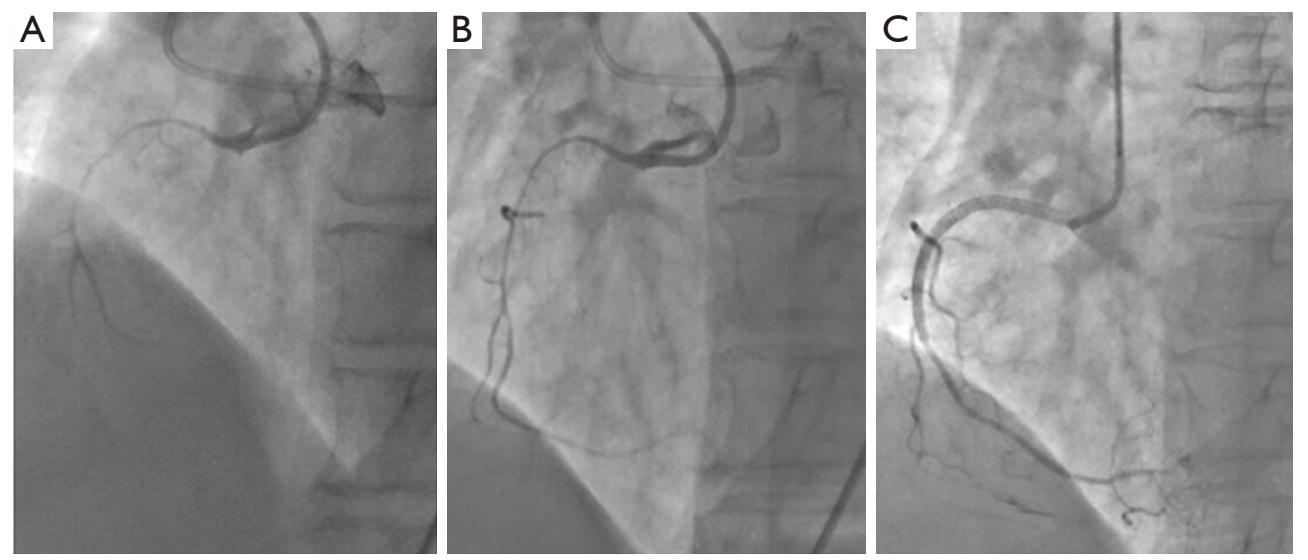

Figure 5 Coronary angiography showed the process of percutaneous coronary angioplasty (PCI) operation to open the occluded right coronary artery (RCA).

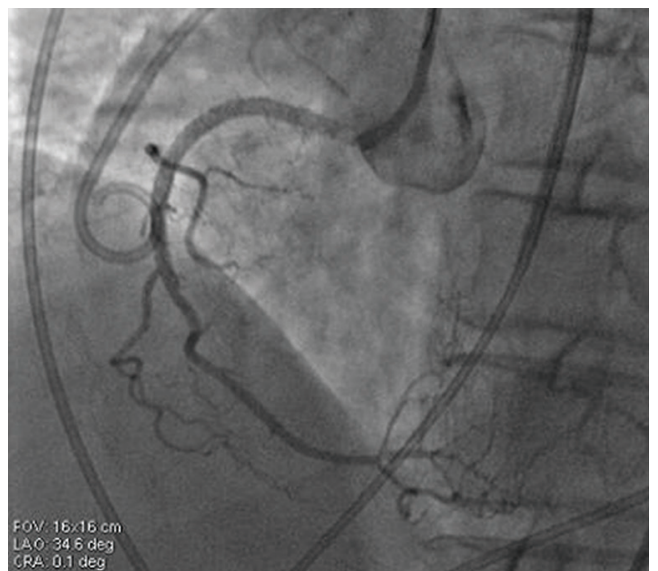

Figure 6 Coronary angiography shows no the rupture of the coronary artery after percutaneous coronary angioplasty (PCI).

the liquid area of pericardial cavity in the posterior wall of the left ventricle was $10 / 7 \mathrm{~mm}(\mathrm{SD})$, that of the anterior wall of right ventricle was $5 / 3 \mathrm{~mm}$ (SD), and that of the pericardial cavity of the right apex was $10 / 6 \mathrm{~mm}(\mathrm{~S} / \mathrm{D})$. Pericardial effusion is between the low and moderate level. Right coronary angiography showed that the blood flow of RCA was normal. There was no contrast agents leaking, but the patient suffered the pericardial effusion. The blood flow of the RCA stent was normal, and there was no loss of lumen or retention of contrast agent (Figure 6). Pericardial puncture was then performed, and about $670 \mathrm{~mL}$ of blood was drained from the pericardium. In order to maintain blood pressure, the patient received intravenous drip infusion of dopamine hydrochloride, noradrenaline bitartrate and transfusion of $800 \mathrm{~mL}$ red blood cells. After assessing the patient's condition, the attending physician decided to transfer the patient to the department of cardiovascular surgery for thoracotomy. After opening pericardium, a large amount of bleeding was cleared and the blood pressure was increased up to $121 / 68 \mathrm{mmHg}$. On the surface of RCA, the hematoma at the distal end of the RCA was detected, but there was no significant bleeding. Hemostasis was carried out on the surface of coronary artery by cardiac surgery. After surgery, the patient continued to take aspirin, clopidogrel and atorvastatin and she was discharged without any symptoms. Three months later, she was admitted to our department for a check, and the echocardiogram showed she recovered well. After 2-year follow-up, the ECG showed T wave inversion in V1-V6, aVL leads (Figure 7) while the patient felt well.

All procedures performed in studies involving human participants were in accordance with the ethical standards of the institutional and/or national research committee(s) and with the Helsinki Declaration (as revised in 2013). Written informed consent was obtained from the patient.

\section{Discussion}

CTO refers to the gradual occlusion of chronic coronary artery (4). CTO can cause insufficient blood supply to the myocardium, and lead to clinical symptoms (5). Many studies confirm that the revascularization of CTO (CTOPCI) is associated with reducing the incidence of angina pectoris and improving heart function and the quality of life (6). However, there were some serious complications 


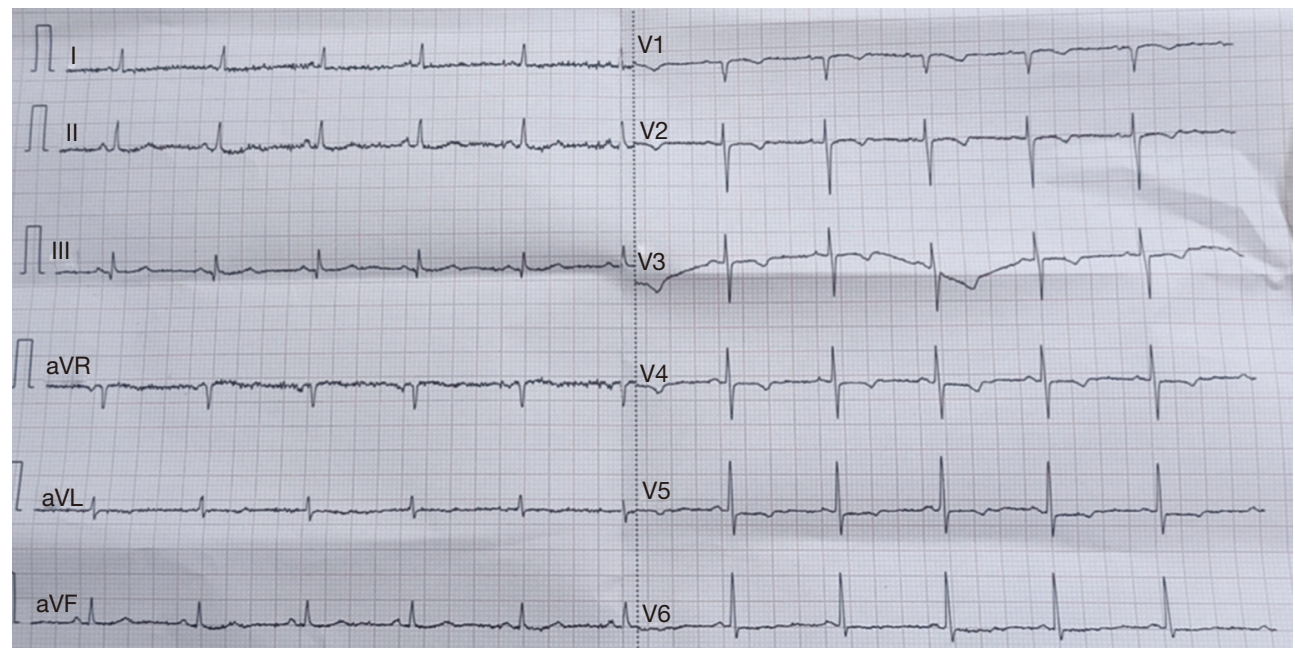

Figure 7 Electrocardiogram (ECG) showed ST depression and T wave inversion in V1-V6, I, AVL leads.

in CTO-PCI procedure, for example the coronary artery rupture. Coronary artery rupture is one of the most worrying complications in CTO-PCI $(7,8)$, because it may lead to more dangerous clinical conditions such as pericardial tamponade, which causes hypotension and shock, eventually resulting to death.

Usually, coronary angiogram is carried out to determine the rupture spot of the coronary artery (9). In our case, there was no contrast agent leaking from coronary arteries. But the consistently increased pericardial effusion led to decreased blood pressure. We faced confusion and difficulty of treatment. Then, we transferred the patient to Cardiac Surgery Department. The hematoma was found under the direct vision after thoracotomy and no definite rupture of coronary vessels was detected.

The patient had pericardial tamponade but coronary angiogram was normal. The reason may be as follow. Firstly, we used those hard and slipper wires such as XT$\mathrm{R}$, Gaia wire to revascularize. During the procedure, the wire may go through between the intima and the outer elastic membrane of the blood vessel, then we used balloons and stents to expand, which may lead to the increased permeability of the blood vessel. Secondly, there may be small amount of bleeding at the distal end of the coronary artery where a blood clot formed. The blood clot stopped the bleeding from the rupture of the vessel. However, in the certain limited angiography posture, we may miss the bleeding sites. Thirdly, the diameter of vascular wall leakage spot maybe bigger than that of red blood cell, but smaller than that of contrast agent. Therefore, there was pericardial effusion, but we didn't find contrast agent leaking from coronary artery.

This case indicated that there was still the possibility of coronary artery rupture, although the coronary angiogram showed no rupture of the coronary artery after PCI. We could use IVUS examining coronary artery after vascularization. Maybe IVUS could find the rupture spot of coronary artery. And the symptoms of patients must be observed after the PCI operation.

During follow up, we found the changes of the ECG. After 6 weeks of LCX PCI procedure, the ST stage depressed in V1-V5 leads. And the ST depression and $\mathrm{T}$ inversion in V1-V6, I, aVL leads were found 2 years after CTO revascularization. Actually, we didn't know why the changes of ECG. Maybe we still need the nuclide imaging of myocardial inspect. Maybe probably due to the microvascular occlusion during the PCI procedure $(10,11)$.

\section{Conclusions}

In conclusion, coronary angiogram was performed to make sure the rupture of coronary artery in our report, and it was not completely accurate. The combined use of IVUS and coronary angiogram may improve the accuracy of vascular rupture and the safety of PCI procedure.

\section{Acknowledgments}

Funding: This work was supported by the National Nature Science Foundation of China (Grant number: 91439126). 


\section{Footnote}

Reporting Checklist: The authors have completed the CARE reporting checklist. Available at https://dx.doi. org/10.21037/apm-20-2455

Conflicts of Interest: All authors have completed the ICMJE uniform disclosure form (available at https://dx.doi. org/10.21037/apm-20-2455). The authors have no conflicts of interest to declare.

Ethical Statement: The authors are accountable for all aspects of the work in ensuring that questions related to the accuracy or integrity of any part of the work are appropriately investigated and resolved. All procedures performed in studies involving human participants were in accordance with the ethical standards of the institutional and/or national research committee(s) and with the Helsinki Declaration (as revised in 2013). Written informed consent was obtained from the patient.

Open Access Statement: This is an Open Access article distributed in accordance with the Creative Commons Attribution-NonCommercial-NoDerivs 4.0 International License (CC BY-NC-ND 4.0), which permits the noncommercial replication and distribution of the article with the strict proviso that no changes or edits are made and the original work is properly cited (including links to both the formal publication through the relevant DOI and the license). See: https://creativecommons.org/licenses/by-nc-nd/4.0/.

\section{References}

1. Hess CN, Rao SV, McCoy LA, et al. Identification of hospital outliers in bleeding complications after percutaneous coronary intervention. Circ Cardiovasc Qual Outcomes 2015;8:15-22.

2. Adusumalli S, Morris M, Pershad A. Pseudo-Pericardial Tamponade From Right Ventricular Hematoma After Chronic Total Occlusion Percutaneous Coronary

Cite this article as: Li J, Zhang M, Kang X, Chen R, Wang F, Chen W, Li X. Pericardial tamponade after chronic total occlusion revascularization: a case report and literature review. Ann Palliat Med 2021;10(7):8506-8511. doi: 10.21037/apm-202455
Intervention of the Right Coronary Artery: Successfully Managed Percutaneously With Computerized Tomographic Guided Drainage. Catheter Cardiovasc Interv 2016;88:86-8.

3. Kinnaird T, Anderson R, Ossei-Gerning N, et al. Legacy Effect of Coronary Perforation Complicating Percutaneous Coronary Intervention for Chronic Total Occlusive Disease: An Analysis of 26807 Cases From the British Cardiovascular Intervention Society Database. Circ Cardiovasc Interv 2017;10:e004642.

4. Koelbl CO, Nedeljkovic ZS, Jacobs AK. Coronary Chronic Total Occlusion (CTO): A Review. Rev Cardiovasc Med 2018;19:33-9.

5. Tajti P, Brilakis ES. Chronic Total Occlusion Percutaneous Coronary Intervention: Evidence and Controversies. J Am Heart Assoc 2018; 7:e006732.

6. Werner GS, Martin-Yuste V, Hildick-Smith D, et al. A randomized multicentre trial to compare revascularization with optimal medical therapy for the treatment of chronic total coronary occlusions. Eur Heart J 2018;39:2484-93.

7. Vakamudi S, Ho N, Cremer PC. Pericardial Effusions: Causes, Diagnosis, and Management. Prog Cardiovasc Dis 2017;59:380-8.

8. Cader FA, Haq MM, Nasrin S, et al. Pericardial tamponade due to haemorrhagic pericardial effusion as a complication of prasugrel: a case report. BMC Cardiovasc Disord 2016;16:162.

9. Hirai T, Nicholson WJ, Sapontis J, et al. A Detailed Analysis of Perforations During Chronic Total Occlusion Angioplasty. JACC Cardiovasc Interv 2019;12:1902-12.

10. Bahrmann P, Figulla HR, Wagner M, et al. Detection of coronary microembolisation by Doppler ultrasound during percutaneous coronary interventions. Heart 2005;91:1186-92.

11. Bahrmann P, Werner GS, Heusch G, et al. Detection of coronary microembolization by Doppler ultrasound in patients with stable angina pectoris undergoing elective percutaneous coronary interventions. Circulation 2007;115:600-8. 